\title{
Functional network connectivity alterations in schizophrenia and depression
}

\author{
Xing-jie Wu ${ }^{a, b, *}$, Ling-Li Zeng ${ }^{b, \dagger}$, Hui Shen ${ }^{b}$, Lin Yuan ${ }^{b}$ Jian Qin $^{b}$, Peng Zhang ${ }^{b}$, and \\ De-wen $\mathrm{Hu}^{\mathrm{b}, *}$
}

${ }^{a}$ Department of Control Engineering, Naval Aeronautical and Astronautical University, Yantai, Shandong 264001, China

${ }^{b}$ College of Mechatronics and Automation, National University of Defense Technology, Changsha, Hunan 410073, China

*Corresponding author: College of Mechatronics and Automation, National University of Defense Technology, Changsha, Hunan 410073, China. dwhu@ nudt.edu.cn

\begin{abstract}
There is a high degree of overlap between the symptoms of major depressive disorder (MDD) and schizophrenia, but it remains unclear whether the similar symptoms are derived from convergent alterations in functional network connectivity. In this study, we performed a group independent component analysis on resting-state functional MRI data from 20 MDD patients, 24 schizophrenia patients, and 43 matched healthy controls. The functional network connectivity analysis revealed that, compared to healthy controls, the MDD and schizophrenia patients exhibited convergent decreased positive connectivity between the left and right fronto-parietal control network and decreased negative connectivity between the left control and medial visual networks. Furthermore, the MDD patients showed decreased negative connectivity between the left control and auditory networks, and the schizophrenia patients showed decreased positive connectivity between the bilateral control and language networks and decreased negative connectivity between the right control and dorsal attention networks. The convergent network connectivity alterations may underlie the common primary control and regulation disorders, and the divergent connectivity alterations may enable the distinction between the two disorders. All of the convergent and divergent network connectivity alterations were relevant to the control network, suggesting an important role of the network in the pathophysiology of MDD and schizophrenia.
\end{abstract}

Keywords: major depressive disorder; schizophrenia; functional MRI; fronto-parietal control network; functional network connectivity.

* These authors contributed equally to this work. 


\section{Introduction}

Major depressive disorder (MDD), a common mental illness, is characterized by a pervasive and persistent low mood accompanied by low self-esteem and anhedonia (Liu et al., 2013b; Zeng et al., 2012). Schizophrenia has been characterized by abnormal social behavior and a failure to understand reality, accompanied by anxiety disorders, major depressive illness or substance use disorder (Buckley et al., 2009). As two of the most severe psychiatric disorders, there is a high degree of overlap between MDD and schizophrenia symptoms. Mulholland (1986) reported that the features of schizophrenia, especially those that are 'negative', show many clinical similarities to the syndrome of depression. Kessler et al. (1994) found that 59\% of patients with schizophrenia met the DSM-III criteria for major or mild depression. The well-documented impairments in the ability to successfully engage in social interactions are similar to some extent in schizophrenia and MDD (Billeke and Aboitiz, 2013; Fiszdon et al., 2013; Ladegaard et al., 2014; Lee et al., 2013; Schilbach et al., 2016a; Schilbach et al., 2016b; Schilbach et al., 2014; Schneider et al., 2012). Billeke and Aboitiz (2013) suggested that schizophrenic patients have some trouble with their interactions with other people and their integration in society, and Ladegaard et al., (2014) reported that first-episode depressed patients experience difficulties in all domains of higher-order social cognition, including theory of mind, social perception and metacognition. Schilbach et al. (2016b) found that MDD and schizophrenia patients exhibited common dysconnectivity patterns as indexed by a significant reduction in functional connectivity between the anterior and posterior default mode network (DMN), which might contribute to the interpersonal difficulties of the two groups.

Functional connectivity investigates temporal statistical dependencies among distant brain regions by means of seed-based analysis or independent component analysis (ICA), and functional network connectivity investigates functional connectivity at the network level. Each network is a component of an ICA, which may consist of several remote regions, and the dynamics of each network are described by the ICA time course of that network. Hence, functional network connectivity reflects statistical dependencies among ICA time courses (Arbabshirani and Calhoun, 2011; Jafri et al., 2008; Jimenez et al., 2016). Recently, resting-state functional magnetic resonance imaging (fMRI) studies have demonstrated altered functional connectivity between intrinsic connectivity networks (ICNs) in both groups of patients (Abbott et al., 2013; Chang et al., 2014; Li et al., 2013; Meda et al., 2009; Wang et al., 2015; Zeng et al., 2014a). Chang et al. (2014) reported that patients with schizophrenia exhibited a stronger interaction between the anterior DMN and left fronto-parietal control network, and Abbott et al. (2013) found that relative to healthy controls, MDD patients showed abnormal functional network connectivity between the posterior DMN and another two functional networks: the dorsal medial prefrontal cortex and left dorsal lateral prefrontal cortex. In addition, alterations of functional network connectivity not only existed in these two disorders but were also widely observed in various other types of neuropsychiatric disorders, e.g., epilepsy (Wei et al., 2015; Widjaja et al., 2013) and Alzheimer's disease (Gomez-Ramirez and Wu, 2014; Greicius et al., 2004; Jacobs et al., 2013).

Various mental illnesses, such as MDD and schizophrenia, display spectrum features, which suggests that similar symptoms may share common pathophysiological mechanisms (Perlis et al., 2011; Prakash and Mitra, 2008). So far, alterations of functional network connectivity in MDD and schizophrenia have been widely investigated independently, but there are few studies on the 
convergent and divergent alterations in functional network connectivity across the two groups relative to healthy controls, which may underlie similar and distinct symptoms, respectively.

In the current study, we performed group ICA on resting-state functional magnetic resonance imaging (fMRI) data from 20 MDD patients, 24 schizophrenic patients, and 43 matched healthy controls. For each subject, eleven intrinsic connectivity networks or sub-networks were obtained, and the functional network connectivity among them was evaluated using the time courses of the corresponding components. Then, two-sample $t$-tests were conducted to examine functional network connectivity alterations in the MDD and schizophrenia patients.

\section{Methods}

\subsection{Participants}

In the present study, participants included 20 MDD patients ( $N=20,9$ females) and 24 schizophrenia patients ( $\mathrm{N}=24,12$ females), which were recruited from outpatient departments and inpatient units at the Second Xiangya Hospital of Central South University, and 43 healthy controls ( $\mathrm{N}=43$, 19 females) that were recruited from the Changsha City area by word-of-mouth advertising and matched to patients with respect to age (MDD, 26.62 \pm 7.66 years; schizophrenia, $25.33 \pm 6.16$ years; control, 28.23 \pm 7.23 years; $p=0.175$, one-way ANOVA), gender (Pearson's Chi-square test, MDD vs. schizophrenia: $p=0.741$, MDD vs. control: $p=0.952$, schizophrenia vs. control: $p=0.647$ ), and education level (MDD, 12.39 \pm 2.25 years; schizophrenia, 12.29 \pm 2.50 years; control, $12.75 \pm 2.82$ years; $p=0.203$, one-way ANOVA). Portions of these data have been previously reported (Liu et al., 2012; Su et al., 2016; Yu et al., 2013).

All subjects were physically healthy as indicated through physical examinations before screening and provided their written informed consent after receiving a complete description of this study. This study was approved by the Ethics Committee of the Second Xiangya Hospital of Central South University. All patients were evaluated by the Structured Clinical Interview for DSM-IV (Diagnostic and Statistical Manual of Mental Disorders, Fourth Edition) (Siegel and Elaine). The MDD and schizophrenia patients met the DSM-IV criteria for MDD and schizophrenia, respectively. The MDD patients had scores on the 17-item Hamilton Depression Rating Scale (HDRS) (Hamilton, 1960) of 25.46 \pm 6.36 , and schizophrenia patients had scores on the Positive and Negative Syndrome Scale (PANSS) (Kay et al., 1987) of 87.14 \pm 12.20 . Six of the patients with schizophrenia were medication-free, while the others accepted atypical psychotropic drugs during the time of scanning (risperidone [n=10, 2-6 mg/day], clozapine [n=4, 200-350 $\mathrm{mg} /$ day], quetiapine [ $\mathrm{n}=4,400-600 \mathrm{mg} /$ day], and sulpiride [ $\mathrm{n}=1,200 \mathrm{mg} / \mathrm{day}])$. All patients with MDD were first-episode, medication-naïve. All patients were abstinent from caffeine, nicotine and alcohol prior to the scanning session. Exclusion criteria for patients included substance abuse or dependence, acute physical illness, a history of head injury resulting in loss of consciousness, and a major psychiatric or neurological illness other than MDD or schizophrenia, and the similar criteria were adopted for healthy control subjects. No subject was excluded due to excessive head motion (translation $<2.5 \mathrm{~mm}$ and rotation $<2$ degree), and there was no significant difference in head motion between groups ( $p>0.10$, one-way ANOVA) (Zeng et al., 2014b).

\subsection{Image acquisition and preprocessing}

Images were acquired on a 1.5-T GE Signa System (GE Signa, Milwaukee, Wisconsin, USA). The parameters were as follows: $\mathrm{TR}=2000 \mathrm{~ms}, \mathrm{TE}=40 \mathrm{~ms}, \mathrm{FA}=90^{\circ}, \mathrm{FOV}=24 \mathrm{~cm}$, matrix $=$ 
$64 \times 64$, slices $=20$, gap $=1 \mathrm{~mm}$, and slices thickness $=5 \mathrm{~mm}$. Subjects were instructed to stay awake, keep their eyes closed, minimize head movement and perform no specific cognitive exercise. Each fMRI session lasted 6 minutes, obtaining 180 volumes.

Data preprocessing was performed using the statistical parametric mapping software package (SPM8, Welcome Department of Cognitive Neurology, Institute of Neurology, London, UK, http://www.fil.ion.ucl.ac.uk/spm). Due to magnetic saturation effects, the first five volumes of each subject were discarded. In the remaining volumes, head motion correction, spatial normalization (using the standard echo planar imaging template in the Montreal Neurological Institute space), spatial smoothing (using a Gaussian filter of an 8-mm full-width half-maximum kernel), and temporal filtering (using a Chebyshev band-pass filter (0.01-0.08 Hz)) were successfully performed.

\subsection{Group independent component analysis}

In the present study, group spatial ICA (Calhoun et al., 2001) was performed on all 87 participants using the fast ICA algorithm (Hyvärinen and Oja, 1997). Because of its ability to discover spatially independent components without any hypothesis or prior knowledge, this method has been widely used in the analysis of resting-state fMRI images (Beckmann et al., 2005). Here, data were decomposed into thirty components using the GIFT software (http://icatb.sourceforge.net/, version 1.3e). Dimension estimation to determine the number of components was performed using the minimum description length criteria (Jafri et al., 2008). Single subject time courses and spatial maps were then computed (back-reconstruction), during which the aggregate components and the results from the data reduction were used to compute the individual subject components.

\subsection{Functional network connectivity analysis}

A systematic process was utilized to select the functional network of interest (Stevens et al., 2007). The association of each component's spatial map with a priori probabilistic maps of gray matter, white matter, and cerebral spinal fluid (CSF) within standardized brain space (MNI templates in SPM8) helped to identify those components whose patterns of correlated signal change largely comprised gray matter versus non-gray matter. Components with high correlation to a priori localized CSF or white matter or with low correlation to gray matter were thought to be artifactual rather than representing hemodynamic change. Visual inspection of discarded components suggested that they represented eye movements, head motion, or cardiac-induced pulsatile artifact at the base of the brain (Jafri et al., 2008). According to previous studies (Chang et al., 2014; Laird et al., 2011; Liu et al., 2013a; Smith et al., 2009; Wei et al., 2015; Yeo et al., 2011; Zeng et al., 2012), eleven independent components were selected, including the posterior and anterior DMN, the left and right fronto-parietal control network, the primary visual network, the medial visual network, the auditory network, the language network, the sensorimotor network, and the ventral and dorsal attention network (ATN).

Functional network connectivity was measured by Pearson correlation coefficients between the eleven networks or sub-networks, and the coefficients were converted to z-scores using the Fisher r-to-z transformation. Thus, for each subject, $55(11 \times 10 / 2)$ z-values of functional connectivity could be obtained. For each connection, we applied ANOVA analysis across 87 subjects of three groups. The functional network connections with significant difference $(p<0.05)$ across the three groups in the ANOVA analysis were gathered together. In these significantly 
different connections, pairwise two-sample t-tests $(p<0.05$, FDR corrected) were performed on three groups to assess the specific group differences. The F, df, p value of the ANOVA analysis are listed in the Supplemental Table 1 and the means, standard deviations of 55 network connection strengths of three groups are presented in Supplemental Table 2-4.

\section{Results}

\subsection{Independent components}

The spatial maps and corresponding time courses (the red signal below each map, named as scans) of the eleven networks or sub-networks are presented in Fig. 1. The spatial maps were obtained by one-sample $t$-tests ( $p<0.05$, FDR corrected): (a), the posterior DMN, including the posterior cingulate cortex, precuneus cortex, and bilateral angular gyri; (b), the anterior DMN, including the anterior and middle cingulate cortex, medial prefrontal cortex, and a part of the precuneus cortex; (c), the left fronto-parietal control network, including the left dorsolateral prefrontal cortex (DLPFC), left inferior parietal lobe (IPL) and middle temporal gyrus (MTG); (d), the right fronto-parietal control network, including the right DLPFC, right IPL and MTG; (e), the primary visual network, including the primary visual cortex; (f), the medial visual network, including the cuneus, posterior precuneus, lingual gyrus, and a part of the fusiform gyrus; $(\mathrm{g})$, the auditory network, including the superior temporal gyrus, Heschl's gyrus, the operculum, middle and posterior insula, and a part of the cuneus; (h), the language network, including Wernicke's area, Broca's area, the left dorsolateral prefrontal cortex, and left medial superior frontal cortex including the anterior cingulate gyrus; (i), the sensorimotor network, including the precentral cortex, postcentral cortex, anterior region of the parietal lobe, and posterior region of the superior frontal gyrus; (j), the ventral ATN, including the temporoparietal junction (TPJ), the ventral frontal cortex (VFC), middle temporal gyrus, and the lingual gyrus; (k) the dorsal ATN, including the intraparietal sulcus (IPS), frontal eye fields (FEF), and lateral visual cortex.

\subsection{Convergent functional network connectivity}

Compared with healthy controls, the MDD and schizophrenia patients showed convergent decreased positive functional network connectivity between the left and right fronto-parietal control network and convergent decreased negative functional network connectivity between the left fronto-parietal control network and medial visual network (two-sample $t$-tests, $p<0.05$, FDR corrected).

\subsection{Divergent functional network connectivity}

The MDD and schizophrenia patients presented divergent functional network connectivity compared with that in healthy controls (two-sample $t$-tests, $p<0.05$, FDR corrected): the MDD patients showed decreased negative functional network connectivity between the left fronto-parietal control network and auditory network; the schizophrenia patients showed decreased positive functional network connectivity between the bilateral fronto-parietal control network and language network and decreased negative functional network connectivity between the right fronto-parietal control network and dorsal ATN. Relative to the schizophrenia patients, the MDD patients presented decreased negative functional network connectivity between the left fronto-parietal control network and the auditory network and increased positive functional 
network connectivity between the right fronto-parietal control network and the language network (two-sample $t$-tests, $p<0.05$, FDR corrected). These results are shown in Fig. 2, Fig. 3 and Fig. 4. The bars in Fig. 2 are to illustrate the means and standard deviations of network connection strengths and Fig. 2 is visualized into Fig. 3 and Fig. 4.

\section{Discussion}

In the present study, we performed group ICA on resting-state fMRI data from three groups: MDD patients, schizophrenia patients, and matched healthy controls, and eleven intrinsic connectivity networks or sub-networks were obtained. In the functional network connectivity analysis, we observed that, compared with healthy controls, the MDD and schizophrenia groups exhibited convergent decreased positive functional network connectivity between the left and right fronto-parietal control network and decreased negative functional network connectivity between the left fronto-parietal control network and the medial visual network. Furthermore, the MDD patients showed decreased negative functional network connectivity between the left fronto-parietal control network and the auditory network, and the schizophrenia patients showed decreased positive functional network connectivity between both sides of the fronto-parietal control network and the language network and decreased negative functional network connectivity between the right fronto-parietal control network and the dorsal attention network. Relative to schizophrenia patients, the MDD patients presented decreased negative functional network connectivity between the left fronto-parietal control network and the auditory network and increased positive functional network connectivity between the right fronto-parietal control network and the language network.

The fronto-parietal control network mainly contains the bilateral dorsolateral prefrontal cortex (DLPFC), inferior parietal lobe (IPL) and middle temporal gyrus (MTG) (Chang et al., 2014). The fronto-parietal control network is often evoked by various cognitive tasks (Cole et al., 2013b; Dosenbach et al., 2007; Dosenbach et al., 2006; Fassbender et al., 2010; Fox et al., 2005; Vincent et al., 2008) and acts as a medial controller for the interaction of the networks in the brain (Chang et al., 2014; Wei et al., 2015). In several mental illness, multiple executive functions driven by the fronto-parietal control network are impaired (Chang et al., 2014; Torrey, 2007; Woo et al., 2008; Woodward et al., 2011; Yildiz et al., 2011). Cole et al. (2014) proposed that the fronto-parietal control system, of which the fronto-parietal control network was one main component, played a central role in promoting and maintaining mental health and implemented feedback control to regulate the symptoms of mental disorders as they arose. They considered an intact fronto-parietal control system to be protective against a variety of mental diseases. According to the studies of Cole and colleagues, the fronto-parietal control system consisted of the fronto-parietal control network, cingulo-opercular network, and dorsal ATN. Evidence for alterations of the fronto-parietal control system has been well established in MDD (Lee and Hermens, 2012; Zhang et al., 2011) and schizophrenia (Anticevic et al., 2012; Barch and Ceaser, 2012; Cole et al., 2011). All of the convergent and divergent functional network connectivity alterations found in this study were relevant to the fronto-parietal control network, as shown in Fig. 3 and Fig. 4. In light of these findings, we suggested that the dysfunction of the fronto-parietal control network was an important mechanism underlying the pathophysiology of MDD and schizophrenia.

To further elucidate its role in various types of mental disorders, the fronto-parietal control network is often investigated as two subsystems: the left and the right fronto-parietal control 
network. Rotarska-Jagiela et al. (2010) reported that, within the fronto-parietal control network, patients with schizophrenia showed decreased separation between the left and right fronto-parietal control network, with the right fronto-parietal control network laterality index correlating with disorganization symptom severity. Kohler et al. (1998) found that frontal lobe dysfunction may result in the depressive symptom in schizophrenia. Other studies have also provided presumptive evidence for the common pathology of schizophrenia and MDD, particularly in the prefrontal cortex (Bearden et al., 2001; Buchanan et al., 1998; Buchsbaum and Hazlett, 1998). In certain neuropsychiatric conditions (e.g., MDD and schizophrenia), fronto-parietal control system disruption is itself one of the primary pathophysiological processes of the disease (Cole et al., 2014). Such primary control disorders involving the neural dysfunction of the control system (e.g., abnormal functional network connectivity between the left and right fronto-parietal control network) are crucial and especially harmful to the psychology of MDD and schizophrenia, as are the immunodeficiency diseases to the body. The visual network is involved in visual information processing. The medial visual network mainly consists of the cuneus, posterior precuneus and lingual gyrus, and part of the fusiform gyrus. Zeng et al. (2012) suggested that the altered functional connectivity related to the fusiform gyrus as a portion of the medial visual network, which is involved in the perception of emotions in facial stimuli, might account for the social avoidance observed in depressed patients. Avsar et al. (2011) showed that schizophrenia presented decreased activation in visual processing areas during the encoding and discrimination phases. In regards to the convergent functional network connectivity alterations, our studies were consistent with these previous findings to some extent. Buschman et al. (2011) suggested that the cognitive capacity of adult humans has a severe limitation, and the originally limited capacity is reduced in neuropsychiatric diseases. The cognitive capacity is not only limited but also shared by varieties of functional networks, and the flexible hubs that exist in the fronto-parietal control system regulate the originally limited and shared capacity via task-dependent biases of their connectivity with other functional networks (Buschman et al., 2011; Cole et al., 2013a; Cole et al., 2013b). We proposed that the decreased negative functional network connectivity between the left fronto-parietal control network and the medial visual network was a type of regulation disorder. Accordingly, these convergent functional network connectivity alterations might reveal some convergent pathological mechanisms of MDD and schizophrenia, which consist of convergent primary control and regulation disorders.

Patients with MDD show impairments in cognitive functions, most of which seem to be related to auditory processing. The auditory network is associated with speech execution, language paradigms and auditory perception (Hackett, 2011; Janssen et al., 2013; Maudoux et al., 2012). Christ et al. (2008) reported that depression in patients was accompanied by cortical dysfunction, including impaired auditory processing of non-speech stimuli. Kahkonen et al. (2007) found that early auditory processing was impaired in patients with MDD during an acute episode, which probably reflected dysfunctional fronto-temporal neural circuits. In the present study, decreased negative functional network connectivity between the left fronto-parietal control network and the auditory network was observed in MDD patients, which was in line with the previous studies. As discussed above, we attributed this altered functional network connectivity to a type of regulation disorder.

There are two vital areas for the language network: Wernicke's area (BA 22 with some branches extending around the posterior section of the lateral sulcus in the parietal lobe) and Broca's area (BA 44 and 45), which are usually located in the dominant hemisphere (the left 
hemisphere for 97\% population) (Snell, 2011). Furthermore, Tomasi and Volkow (2012) found that the neural language loop involved not only the Broca's and Wernicke's area but also the adjoining prefrontal cortex. The language network defined by Tie et al. (2014), which was obtained from group ICA and contained most of superior/middle/inferior frontal gyrus along with part of superior/middle temporal gyrus of the dominant hemisphere, has a high coincidence with our results in the present study. Schizophrenia, which is characterized by language disorders (Leroux et al., 2014), had been suggested to be "the price that Homo sapiens pay for language" (Crow, 1997). Ketteler and Ketteler (2010) proposed that hemispheric imbalance of language processing might play a crucial role in the development of schizophrenia. Li et al. (2012) reported that aberrant systems-level topology of the language processing network, especially significantly weakened network hubs in the left inferior frontal and right fusiform gyrus, might serve as a candidate biomarker of schizophrenia. In our experimental results, decreased positive functional network connectivity between the language network and bilateral fronto-parietal control network was observed in schizophrenic patients, which represents one type of regulation disorder. Accordingly, we speculated that the decrease in the control force of the fronto-parietal control network on the language network might be a critical factor accounting for the behavioral symptoms of schizophrenia.

The dorsal ATN mainly incorporates the IPS and FEF and is also a part of the fronto-parietal control system, which plays a central role in maintaining mental health (Cole et al., 2014; Corbetta and Shulman, 2002; Vossel et al., 2014). Therefore, the decreased negative functional network connectivity between the dorsal ATN and right fronto-parietal control network also reflected the disruption of the control system itself (primary control disorder). Attention deficits were prominent in schizophrenia (Woodward et al., 2011). (Jimenez et al. (2016))reported that when attention demands were increased during a dual-target task, schizophrenic patients showed over-activation of the posterior intra-parietal cortex, a key region of the dorsal ATN. Hence, we deduced that the decreased negative functional network connectivity between the dorsal ATN and the right fronto-parietal control network found in our experiment might reflect decreased inhibition of the right fronto-parietal control network on the dorsal ATN.

Functional network connectivity between the left fronto-parietal control network and the language network and between the right fronto-parietal control network and the dorsal ATN did not show a significant difference between MDD and schizophrenia patients. As presented in Fig. 2 , the mean $\mathrm{z}$-scores of both of these functional connections in MDD patients were located between those of control subjects and schizophrenia patients. Generally, these divergent functional network connectivity alterations may enable the distinction between the two patient groups on the basis of brain imaging. Generally, we suggested that one of the most essential differences between MDD and schizophrenia patients was the divergent alterations in functional network connectivity with the fronto-parietal control network because of the central role of the fronto-parietal control network in the two types of mental diseases. In other words, the specific combination of functional network connectivity alterations with the fronto-parietal control network represented a certain collection of primary control and regulation disorders and determined the mental disease group (MDD or schizophrenia) to which the individuals belonged.

There are several limitations that should be considered in the present study. First, the clinical correlation analysis between the altered functional network connectivity and clinical features might provide some face validity for differences between the patient groups, but the small sample size may limit such analyses, and thus we did not include them in the current study. Second, some 
patients in the present study were receiving antipsychotic medications at the time of scanning that could influence resting-state functional network connectivity activity. This issue should be addressed in the future, if enough data of drug-naïve schizophrenia patients are collected. Third, the MRI scanner with 1.5-Tesla field strength was used in this study. It is possible that the main magnetic field strength of the scanner might affect the power to detect disease effects. The current results should be confirmed by using scanners with higher magnetic field strength in the future. Finally, patients with schizophrenia were not scored on the HDRS, so it is not possible to confirm how many individuals met the criteria for both diseases.

\section{Conclusion}

The current study revealed that, compared to healthy controls, the MDD and schizophrenia groups exhibited convergent decreased positive functional network connectivity between the left and right fronto-parietal control network and decreased negative functional network connectivity between the left fronto-parietal control network and the medial visual network, perhaps underlying some common pathological mechanisms of the two disorders. In addition, the MDD patients showed decreased negative functional network connectivity between the left fronto-parietal control network and the auditory network, and the schizophrenia patients showed decreased positive functional network connectivity between the bilateral fronto-parietal control network and the language network and decreased negative functional network connectivity between the right fronto-parietal control network and the dorsal attention network. These divergent functional network connectivity alterations may enable the distinction between the two disorders on the basis of brain imaging. All the findings suggest a potential important role of the fronto-parietal control network in the pathophysiology of MDD and schizophrenia.

\section{Acknowledgements}

This work was supported by the National Science Foundation of China (61503397, 61420106001, 61375111, and 9142030002) and the National Basic Research Program of China (2013CB329401).

\section{References}

Abbott, C.C., Lemke, N.T., Gopal, S., Thoma, R.J., Bustillo, J., Calhoun, V.D., Turner, J.A., 2013. Electroconvulsive therapy response in major depressive disorder: a pilot functional network connectivity resting state fMRI investigation. Frontiers in Psychiatry 4, 10-10.

Anticevic, A., Repovs, G., Krystal, J.H., Barch, D.M., 2012. A broken filter: Prefrontal functional connectivity abnormalities in schizophrenia during working memory interference. Schizophrenia Research 141, 8-14.

Arbabshirani, M.R., Calhoun, V.D., 2011. Functional network connectivity during rest and task: comparison of healthy controls and schizophrenic patients, Conference: International Conference of the IEEE Engineering in Medicine \& Biology Society IEEE Engineering in Medicine \& Biology Society Conference, pp. 4418-4421. Avsar, K.B., Stoeckel, L.E., Bolding, M.S., White, D.M., Tagamets, M.A., Holcomb, H.H., Lahti, A.C., 2011. Aberrant visual circuitry associated with normal spatial match-to-sample accuracy in schizophrenia. Psychiatry Research 193, 138-143. 
Barch, D.M., Ceaser, A., 2012. Cognition in schizophrenia: core psychological and neural mechanisms. Trends in Cognitive Sciences 16, 27-34.

Bearden, C.E., Hoffman, K.M., Cannon, T.D., 2001. The neuropsychology and neuroanatomy of bipolar affective disorder: a critical review. Bipolar Disorders 3, 106-150.

Beckmann, C.F., Deluca, M., Devlin, J.T., Smith, S.M., 2005. Investigations into resting-state connectivity using independent component analysis. Philosophical Transactions of the Royal Society of London 360, 1001-1013.

Billeke, P., Aboitiz, F., 2013. Social cognition in schizophrenia: from social stimuli processing to social engagement. Frontiers in Psychiatry 4.

Buchanan, R.W., Vladar, K., Barta, P.E., Pearlson, G.D., 1998. Structural evaluation of the prefrontal cortex in schizophrenia. American Journal of Psychiatry 155, 1049-1055.

Buchsbaum, M.S., Hazlett, E.A., 1998. Positron emission tomography studies of abnormal glucose metabolism in schizophrenia. Schizophrenia Bulletin 24, 343-364.

Buckley, P.F., Miller, B.J., Lehrer, D.S., Castle, D.J., 2009. Psychiatric comorbidities and schizophrenia.

Schizophrenia Bulletin 35, 383-402.

Buschman, T.J., Siegel, M., Roy, J.E., Miller, E.K., 2011. Neural substrates of cognitive capacity limitations. Proceedings of the National Academy of Sciences of the United States of America 108, 11252-11255.

Calhoun, V.D., Adali, T., Pearlson, G.D., Pekar, J.J., 2001. A method for making group inferences from functional MRI data using independent component analysis. Human Brain Mapping 14, 140-151.

Chang, X., Shen, H., Wang, L., Liu, Z., Xin, W., Hu, D., Miao, D., 2014. Altered default mode and fronto-parietal network subsystems in patients with schizophrenia and their unaffected siblings. Brain Research 1562, 87-99.

Christ, M., Michael, N., Hihn, H., Schüttke, A., Konrad, C., Baune, B.T., Jansen, A., Pfleiderer, B., 2008. Auditory processing of sine tones before, during and after ECT in depressed patients by fMRI. Journal of Neural Transmission 115, 1199-1211.

Cole, M.W., Anticevic, A., Repovs, G., Barch, D., 2011. Variable globaldysconnectivity and Individual differences in schizophrenia. Biological Psychiatry 70, 43-50.

Cole, M.W., Laurent, P., Stocco, A., 2013a. Rapid instructed task learning: a new window into the human brain's unique capacity for flexible cognitive control. Cognitive Affective \& Behavioral Neuroscience 13, 4840-4849. Cole, M.W., Repovš, G., Anticevic, A., 2014. The frontoparietal control system: a central role in mental health. The Neuroscientist : a review journal bringing neurobiology, neurology and psychiatry 20, 652-664.

Cole, M.W., Reynolds, J.R., Power, J.D., Repovs, G., Anticevic, A., Braver, T.S., 2013b. Multi-task connectivity reveals flexible hubs for adaptive task control. Nature Neuroscience 16, 1348-1355.

Corbetta, M., Shulman, G.L., 2002. Control of goal-directed and stimulus-driven attention in the brain. Nature Reviews Neuroscience 3, 201-215.

Crow, T.J., 1997. Is schizophrenia the price that Homo sapiens pays for language? Schizophrenia Research 28 , 127-141.

Dosenbach, N.U.F., Fair, D.A., Miezin, F.M., Cohen, A.L., Wenger, K.K., Dosenbach, R.A.T., Fox, M.D., Snyder, A.Z., Vincent, J.L., Raichle, M.E., 2007. Distinct brain networks for adaptive and stable task control in humans. Proceedings of the National Academy of Sciences of the United States of America 104, 11073-11078.

Dosenbach, N.U.F., Visscher, K.M., Palmer, E.D., Miezin, F.M., Wenger, K.K., Kang, H.C., Burgund, E.D., Grimes, A.L., Schlaggar, B.L., Petersen, S.E., 2006. A core system for the implementation of task sets. Neuron 50, 799-812. Fassbender, C., Simoes-Franklin, C., Murphy, K., Hester, R., Meaney, J., Robertson, I.H., Garavan, H., 2010. The role of a right fronto-parietal network in cognitive control. Journal of Psychophysiology 20, 286-296.

Fiszdon, J.M., Fanning, J.R., Johannesen, J.K., Bell, M.D., 2013. Social cognitive deficits in schizophrenia and their relationship to clinical and functional status. Psychiatry Research 205, 25-29. 
Fox, M.D., Snyder, A.Z., Vincent, J.L., Corbetta, M., Van Essen, D.C., Raichle, M.E., 2005. The human brain is intrinsically organized into dynamic, anticorrelated functional networks. Proceedings of the National Academy of Sciences of the United States of America 102, 9673-9678.

Gomez-Ramirez, J., Wu, J., 2014. Network-based biomarkers in alzheimer's disease: review and future directions. Frontiers in Aging Neuroscience 6, 12.

Greicius, M.D., Srivastava, G., Reiss, A.L., Menon, V., 2004. Default-mode network activity distinguishes Alzheimer's disease from healthy aging: evidence from functional MRI. Proceedings of the National Academy of Sciences of the United States of America 101, 4637-4642.

Hackett, T.A., 2011. Information flow in the auditory cortical network. Hearing Research 271, 133-146.

Hamilton, M., 1960. A rating scale for depression. Journal of neurology, neurosurgery, and psychiatry 23, 56-62.

Hyvärinen, A., Oja, E., 1997. A fast fixed-point algorithm for independent component analysis. Neural Computation 9, 1483-1492.

Jacobs, H.I., Radua, J., Luckmann, H.C., Sack, A.T., 2013. Meta-analysis of functional network alterations in Alzheimer's disease: toward a network biomarker. Neuroscience and biobehavioral reviews 37, 753-765.

Jafri, M.J., Pearlson, G.D., Stevens, M., Calhoun, V.D., 2008. A method for functional network connectivity among spatially independent resting-state components in schizophrenia. Neuroimage 39, 1666-1681.

Janssen, A.L., Boster, A., Patterson, B.A., Abduljalil, A., Prakash, R.S., 2013. Resting-state functional connectivity in multiple sclerosis: An examination of group differences and individual differences. Neuropsychologia 51, 2918-2929.

Jimenez, A.M., Lee, J., Wynn, J.K., Cohen, M.S., Engel, S.A., Glahn, D.C., Nuechterlein, K.H., Reavis, E.A., Green, M.F., 2016. Abnormal ventral and dorsal attention network activity during single and dual target detection in schizophrenia. Frontiers in Psychology 7.

Kahkonen, S., Yamashita, H., H, Suominen, K., Ahveninen, J., Isometsa, E., 2007. Dysfunction in early auditory processing in major depressive disorder revealed by combined MEG and EEG. Journal of Psychiatry \& Neuroscience Jpn 32, 316-322.

Kay, S.R., Fiszbein, A., Opler, L.A., 1987. The positive and negative syndrome scale (PANSS) for schizophrenia. Schizophr Bull 13, 261-276.

Kessler, R.C., Mcgonagle, K.A., Zhao, S., Nelson, C.B., Hughes, M., Eshleman, S., Wittchen, H.U., Kendler, K.S., 1994. Lifetime and 12-month prevalence of DSM-III-R psychiatric disorders in the United States. Results from the National Comorbidity Survey. Archives of General Psychiatry 51, 8-19.

Ketteler, D., Ketteler, S., 2010. Is schizophrenia "the price that Homo sapiens pays for language"? Subcortical language processing as the missing link between evolution and language disorder in psychosis - A neurolinguistic approach. Journal of Neurolinguistics 23, 342-353.

Kohler, C., Gur, R.C., Swanson, C.L., Petty, R., Gur, R.E., 1998. Depression in schizophrenia: I. association with neuropsychological deficits. Biological Psychiatry 43, 165-172.

Ladegaard, N., Larsen, E.R., Videbech, P., Lysaker, P.H., 2014. Higher-order social cognition in first-episode major depression. Psychiatry Research 216, 37-43.

Laird, A.R., Fox, P.M., Eickhoff, S.B., Turner, J.A., Ray, K.L., Mckay, D.R., Glahn, D.C., Beckmann, C.F., Smith, S.M., Fox, P.T., 2011. Behavioral Interpretations of Intrinsic Connectivity Networks. Journal of Cognitive Neuroscience 23, 4022-4037.

Lee, J., Altshuler, L., Glahn, D.C., Miklowitz, D.J., Ochsner, K., Green, M.F., 2013. Social and nonsocial cognition in bipolar disorder and schizophrenia: relative levels of impairment. American Journal of Psychiatry 170, $334-341$. Lee, R., Hermens, D.F., 2012. A meta-analysis of cognitive deficits in first-episode Major Depressive Disorder. Journal of Affective Disorders 140, 113-124.

Leroux, E., Delcroix, N., Dollfus, S., 2014. Left fronto-temporal dysconnectivity within the language network in schizophrenia: An fMRI and DTI study. Psychiatry Research Neuroimaging 223, 261-267. 
Li, B., Liu, L., Friston, K.J., Shen, H., Wang, L., Zeng, L.L., Hu, D., 2013. A treatment-resistant default mode subnetwork in major depression. Biological Psychiatry 74, 48-54.

Li, X., Xia, S., Bertisch, H.C., Branch, C.A., Delisi, L.E., 2012. Unique topology of language processing brain network: a systems-level biomarker of schizophrenia. Schizophrenia Research 141, 128-136.

Liu, F., Guo, W., Fouche, J.P., Wang, Y., Wang, W., Ding, J., Zeng, L., Qiu, C., Gong, Q., Zhang, W., $2013 a$. Multivariate classification of social anxiety disorder using whole brain functional connectivity. Brain Structure \& Function 220, 1-15.

Liu, F., Guo, W., Liu, L., Long, Z., Ma, C., Xue, Z., Wang, Y., Li, J., Hu, M., Zhang, J., 2013b. Abnormal amplitude low-frequency oscillations in medication-naive, first-episode patients with major depressive disorder: a resting-state fMRI study. Journal of Affective Disorders 146, 401-406.

Liu, H., Kaneko, Y., Ouyang, X., Li, L., Hao, Y., Chen, E.Y.H., Jiang, T., Zhou, Y., Liu, Z., 2012. Schizophrenic patients and their unaffected siblings share increased resting-state connectivity in the task-negative network but not its anticorrelated task-positive network. Schizophrenia Bulletin 38, 285-294.

Maudoux, A., Lefebvre, P., Cabay, J.E., Demertzi, A., Vanhaudenhuyse, A., Laureys, S., Soddu, A., 2012. Auditory resting-state network connectivity in tinnitus: A functional MRI study. Plos One 7, 539-539.

Meda, S.A., Stevens, M.C., Folley, B.S., Calhoun, V.D., Pearlson, G.D., 2009. Evidence for anomalous network connectivity during working memory encoding in schizophrenia: an ICA based analysis. Plos One 4, e7911-e7911. Mulholland, C., 1986. The symptom of depression in schizophrenia and its management. Advances in Psychiatric Treatment 27, 169-177.

Perlis, R.H., Uher, R., Ostacher, M., Goldberg, J.F., Trivedi, M.H., Rush, A.J., Fava, M., 2011. Association Between Bipolar Spectrum Features and Treatment Outcomes in Outpatients With Major Depressive Disorder. Archives of General Psychiatry 68, 351-360.

Prakash, J., Mitra, A.K., 2008. Spectrum Disorder: At Newer Horison in Psychiatry. Delhi Psychiatry Journal. Rotarska-Jagiela, A., Van, d.V.V., Oertel-Knöchel, V., Uhlhaas, P.J., Vogeley, K., Linden, D.E., 2010. Resting-state functional network correlates of psychotic symptoms in schizophrenia. Schizophrenia Research 117, 21-30. Schilbach, L., Derntl, B., Aleman, A., Caspers, S., Clos, M., Diederen, K.M.J., Gruber, O., Kogler, L., Liemburg, E.J., Sommer, I.E., Müller, V.I., Cieslik, E.C., Eickhoff, S.B., 2016a. Differential patterns of dysconnectivity in mirror neuron and mentalizing networks in schizophrenia. Schizophrenia Bulletin.

Schilbach, L., Hoffstaedter, F., Müller, V., Cieslik, E.C., Goya-Maldonado, R., Trost, S., Sorg, C., Riedl, V., Jardri, R., Sommer, I., Kogler, L., Derntl, B., Gruber, O., Eickhoff, S.B., 2016b. Transdiagnostic commonalities and differences in resting state functional connectivity of the default mode network in schizophrenia and major depression. NeuroImage: Clinical 10, 326-335.

Schilbach, L., Müller, V.I., Hoffstaedter, F., Clos, M., Goya-Maldonado, R., Gruber, O., Eickhoff, S.B., 2014. Meta-analytically informed network analysis of resting state fMRI reveals hyperconnectivity in an introspective socio-affective network in depression. PLoS ONE 9, e94973.

Schneider, D., Regenbogen, C., Kellermann, T., Finkelmeyer, A., Kohn, N., Derntl, B., Schneider, F., Habel, U., 2012. Empathic behavioral and physiological responses to dynamic stimuli in depression. Psychiatry Research 200 , 294-305.

Siegel, L.J., Elaine, F.J., Diagnostic and statistical manual of mental disorders. American Psychiatric Association. Smith, S.M., Fox, P.T., Miller, K.L., Glahn, D.C., Fox, P.M., Mackay, C.E., Filippini, N., Watkins, K.E., Toro, R., Laird, A.R., 2009. Correspondence of the brain's functional architecture during activation and rest. Proceedings of the National Academy of Sciences of the United States of America 106, 13040-13045.

Snell, R.S., 2011. Neuroanatomia clinica. Wolters/lippincott.

Stevens, M.C., Kiehl, K.A., Pearlson, G., Calhoun, V.D., 2007. Functional neural circuits for mental timekeeping. Human Brain Mapping 28, 394-408. 
Su, J., Shen, H., Zeng, L.L., Qin, J., Liu, Z., Hu, D., 2016. Heredity characteristics of schizophrenia shown by dynamic functional connectivity analysis of resting-state functional MRI scans of unaffected siblings. Neuroreport 27, 843-848.

Tie, Y., Rigolo, L., Norton, I.H., Huang, R.Y., Wu, W., Orringer, D., Jr, M.S., Golby, A.J., 2014. Defining language networks from resting-state fMRI for surgical planning--a feasibility study. Human Brain Mapping 35, $1018-1030$. Tomasi, D., Volkow, N.D., 2012. Resting functional connectivity of language networks: characterization and reproducibility. Molecular Psychiatry 17, 841-854.

Torrey, E.F., 2007. Schizophrenia and the inferior parietal lobule. Schizophrenia Research 97, 215-225.

Vincent, J.L., Kahn, I., Snyder, A.Z., Raichle, M.E., Buckner, R.L., 2008. Evidence for a frontoparietal control system revealed by intrinsic functional connectivity. Journal of Neurophysiology 100, 3328-3342.

Vossel, S., Geng, J.J., Fink, G.R., 2014. Dorsal and ventral attention systems: distinct neural circuits but collaborative roles. The Neuroscientist : a review journal bringing neurobiology, neurology and psychiatry 20 , 150-159.

Wang, H., Zeng, L.L., Chen, Y., Yin, H., Tan, Q., Hu, D., 2015. Evidence of a dissociation pattern in default mode subnetwork functional connectivity in schizophrenia. Scientific Reports 5.

Wei, H.L., An, J., Zeng, L.L., Shen, H., Qiu, S.J., Hu, D.W., 2015. Altered functional connectivity among default, attention, and control networks in idiopathic generalized epilepsy. Epilepsy \& Behavior 46, 118-125.

Widjaja, E., Zamyadi, M., Raybaud, C., Snead, O.C., Smith, M.L., 2013. Abnormal functional network connectivity among resting-state networks in children with frontal lobe epilepsy. AJNR. American journal of neuroradiology 34, 2386-2392.

Woo, T.U., Kim, A.M., Viscidi, E., 2008. Disease-specific alterations in glutamatergic neurotransmission on inhibitory interneurons in the prefrontal cortex in schizophrenia. Brain Res. 1218, 267-277. Brain Research 1218, 267-277.

Woodward, N.D., Rogers, B., Heckers, S., 2011. Functional resting-state networks are differentially affected in schizophrenia. Schizophrenia Research 130, 86-93.

Yeo, B.T., Krienen, F.M., Sepulcre, J., Sabuncu, M.R., Lashkari, D., Hollinshead, M., Roffman, J.L., Smoller, J.W., Zöllei, L., Polimeni, J.R., 2011. The organization of the human cerebral cortex estimated by intrinsic functional connectivity. Journal of Neurophysiology 106, 1125-1165.

Yildiz, M., Borgwardt, S.J., Berger, G.E., 2011. Parietal lobes in schizophrenia: do they matter? Schizophrenia Research \& Treatment 2011, 581686-581686.

Yu, Y., Shen, H., Zeng, L.L., Ma, Q., Hu, D., 2013. Convergent and divergent functional connectivity patterns in schizophrenia and depression. Plos One 8, e68250-e68250.

Zeng, L.L., Shen, H., Liu, L., Hu, D., 2014a. Unsupervised classification of major depression using functional connectivity MRI. Human Brain Mapping 35, 1630-1641.

Zeng, L.L., Shen, H., Liu, L., Wang, L., Li, B., Fang, P., Zhou, Z., Li, Y., Hu, D., 2012. Identifying major depression using whole-brain functional connectivity: a multivariate pattern analysis. Brain 135, 1498-1507.

Zeng, L.L., Wang, D., Fox, M.D., Sabuncu, M., Hu, D., Ge, M., Buckner, R.L., Liu, H., 2014b. Neurobiological basis of head motion in brain imaging. Proceedings of the National Academy of Sciences of the United States of America 111, 6058-6062.

Zhang, J., Wang, J., Wu, Q., Kuang, W., Huang, X., He, Y., Gong, Q., 2011. Disrupted brain connectivity networks in drug-naive, first-episode major depressive disorder. Biological Psychiatry 70, 334-342.

Fig. 1 The spatial maps and corresponding time courses of the eleven components. Fig. 1(a) the posterior default mode network (DMN); Fig. 1(b) the anterior DMN; Fig. 1(c) the left fronto-parietal control network; 
Fig. 1(d) the right fronto-parietal control network; Fig. 1(e) the primary visual network; Fig. 1(f) the medial visual network; Fig. 1(g) the auditory network; Fig. 1(h) the language network; Fig. 1(i) the sensorimotor network; Fig. 1(j) the ventral attention network (ATN); Fig. 1(k) the dorsal ATN.

Fig. 2 The averaged connectivity strengths of significantly different functional network connections among the three groups. The connectivity strength is measured by the z-score. z-score, Fisher r-to-z transformation of Pearson correlation coefficients; MDD, major depressive disorder; IFPN, left fronto-parietal control network; rFPN, right fronto-parietal control network; mVN, medial visual network; AUN, auditory network; LAN, language network; dATN, dorsal attention network.

Fig. 3 Functional network connectivity alterations in major depressive disorder and schizophrenia patients relative to healthy controls (two-sample $t$-tests, $\mathrm{p}<0.05$, FDR corrected). Lines are weighted according to the $p$-value $(-\log (p$-value $) / 2)$ from the $t$-tests; thus, the thicker line indicates that the corresponding alteration is more significant. MDD, major depressive disorder; FPN, fronto-parietal control network; ATN, attention network.

Fig. 4 Functional network connectivity alterations of major depressive disorder patients relative to schizophrenia patients (two-sample $t$-tests, $\mathrm{p}<0.05$, FDR corrected). Lines are weighted according to the $p$-value (-log ( $p$-value) / 2) from the $t$-tests. FPN, fronto-parietal control network; ATN, attention network.

\section{Highlights}

We performed group independent component analysis on resting-state functional magnetic resonance imaging data from major depressive disorder (MDD) patients, schizophrenia patients, and matched healthy controls.

Eleven intrinsic connectivity networks or sub-networks were obtained.

The functional network connectivity (FNC) analysis revealed that MDD and schizophrenia exhibited some convergent and divergent FNC alterations.

The convergent FNC alterations may underlie the common primary control and regulation disorders of MDD and schizophrenia, and the divergent FNC alterations may enable the distinction between the two disorders.

All of the convergent and divergent FNC alterations were relevant to the FPN, suggesting an important role of the FPN in the pathophysiology of MDD and schizophrenia. 

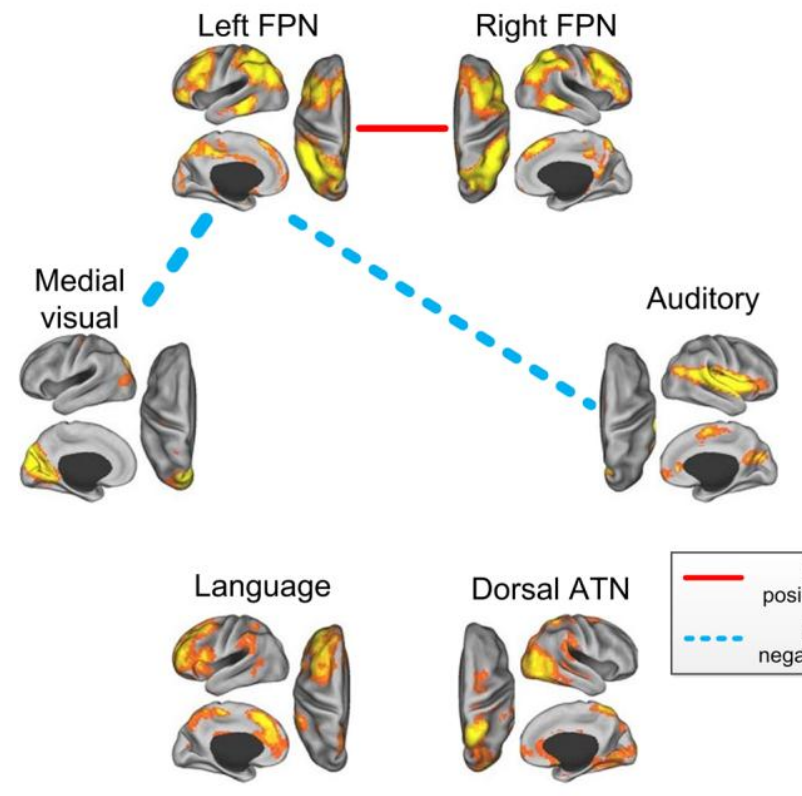

(a) MDD versus control

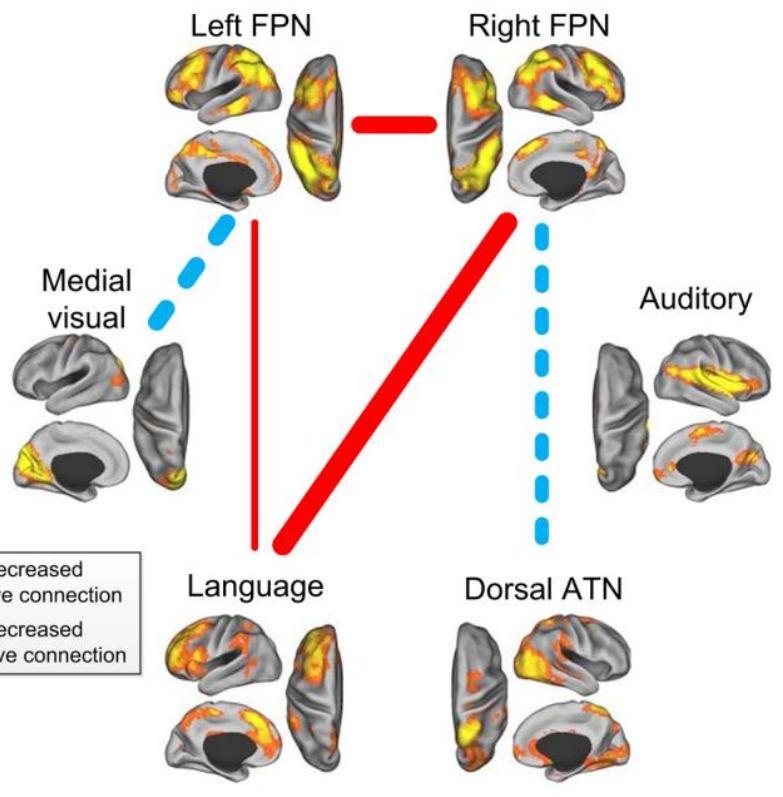

(b) Schizophrenia versus control

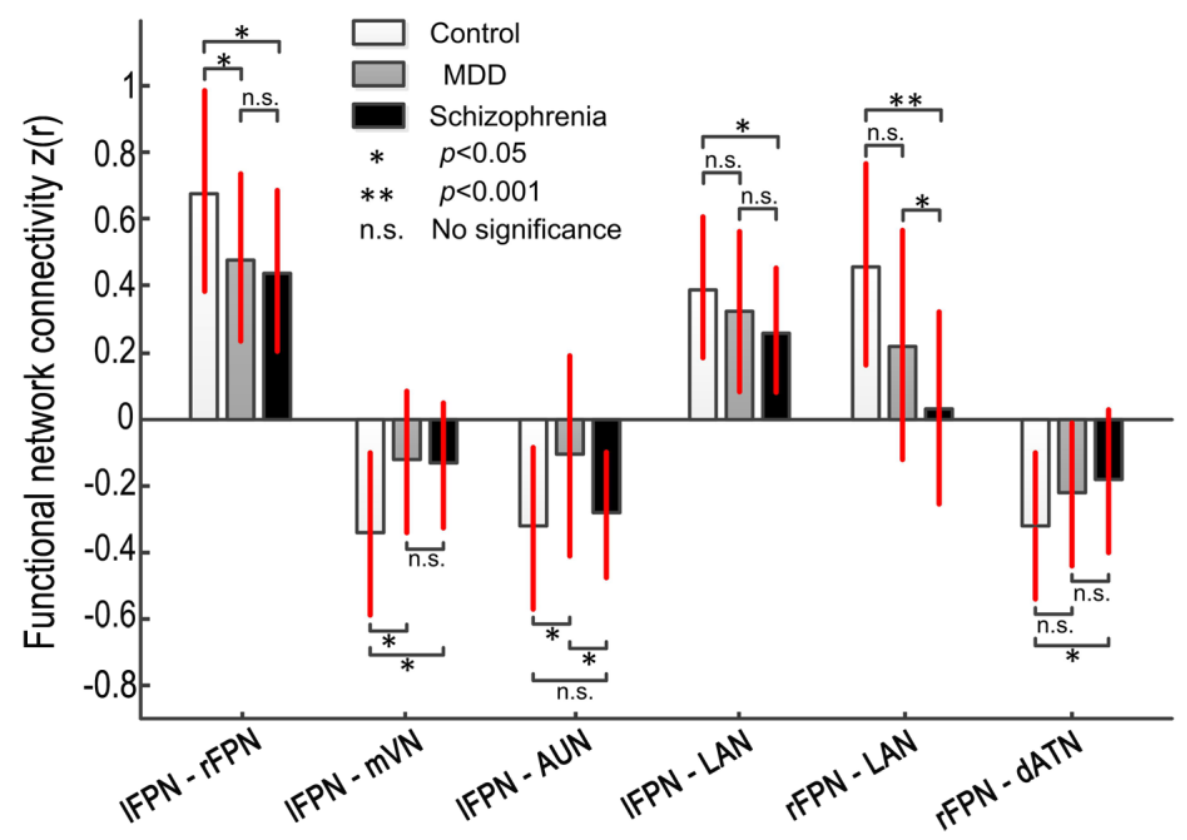


(a) Posterior DMN

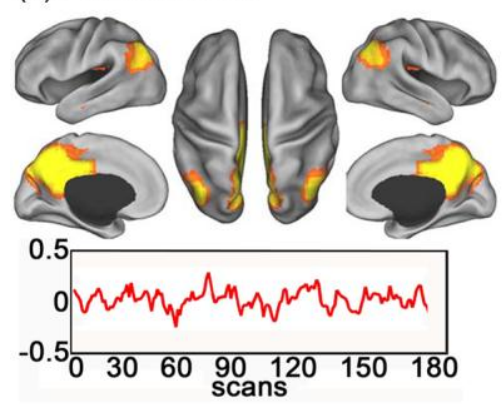

(d) Right FPN

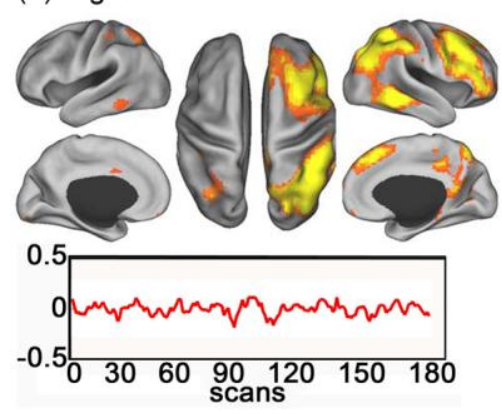

(g) Auditory network

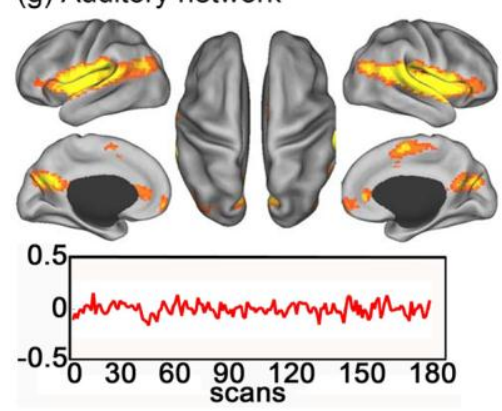

(j) Ventral ATN

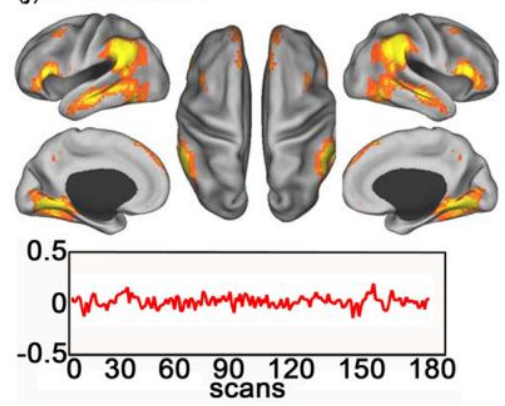

(b) Anterior DMN

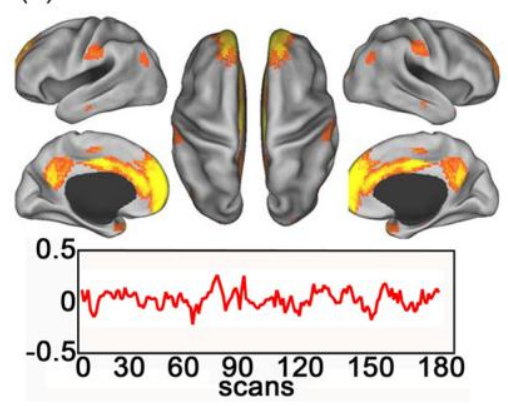

(e) Primary visual network

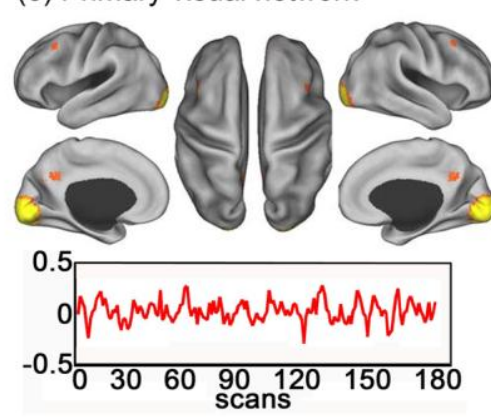

(h) Language network

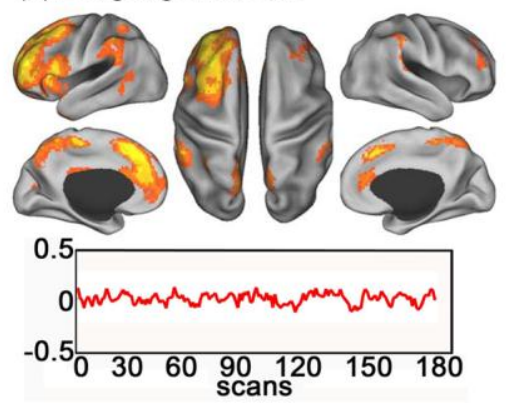

(k) Dorsal ATN

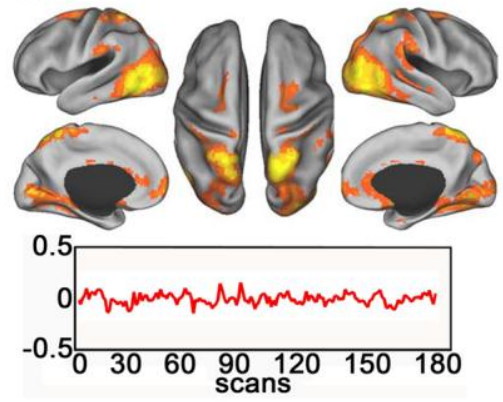

(c) Left FPN

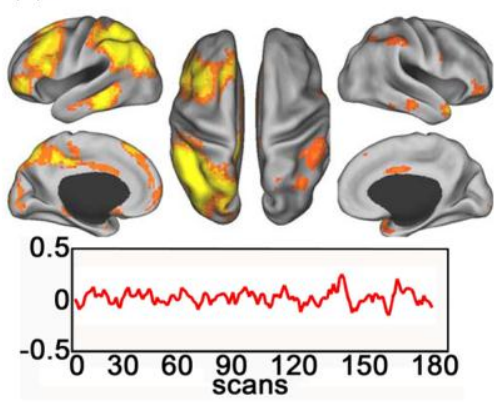

(f) Medial visual network

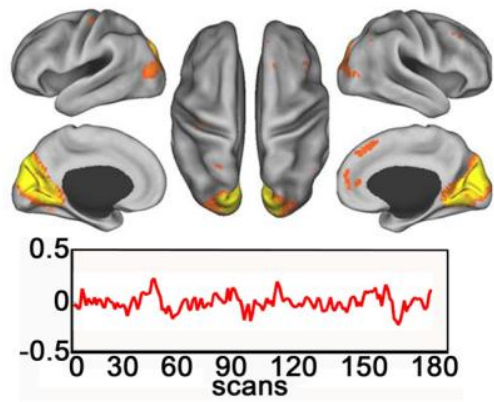

(i) Sensorymotor network

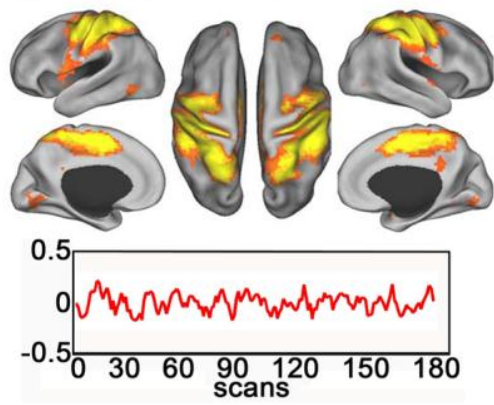

$t$-value

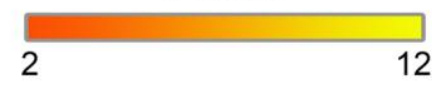




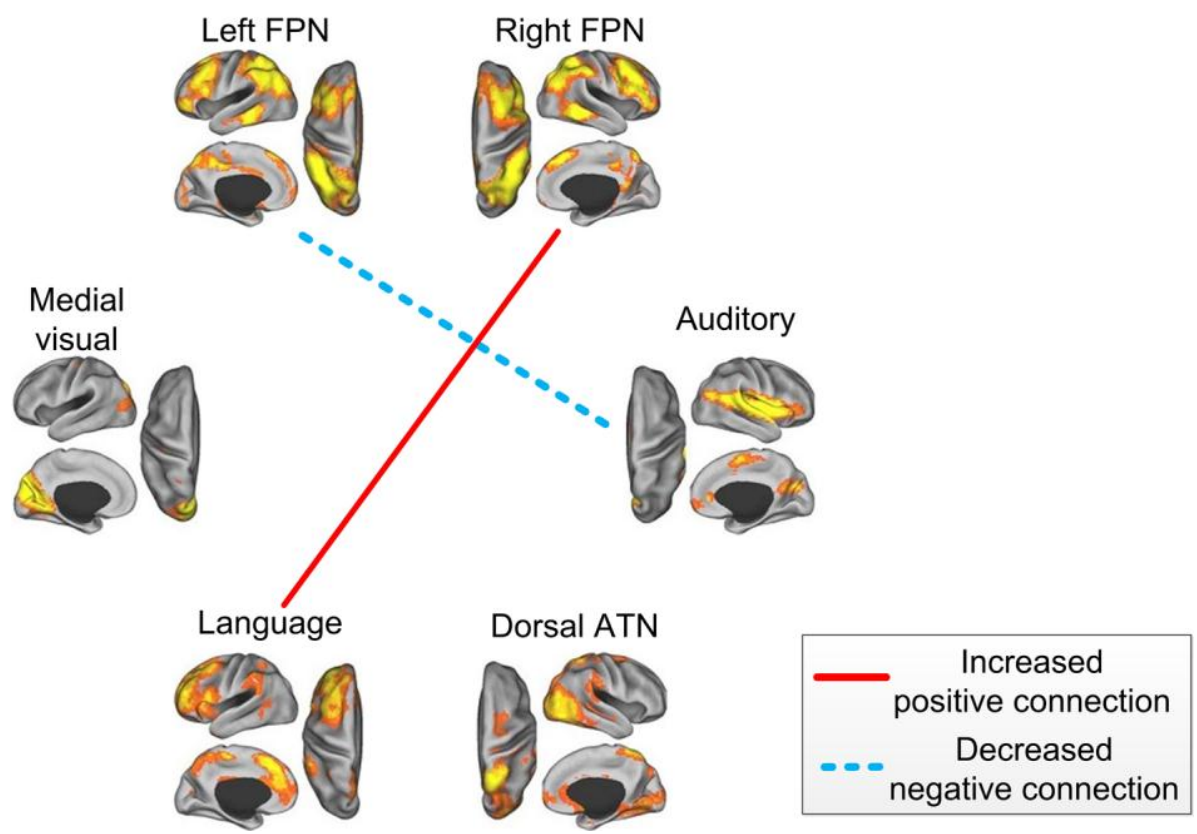

